\title{
REDUCING FALSE ALARM RATES DURING CHANGE DETECTION BY MODELING RELIEF, SHADE AND SHADOW OF MULTI-TEMPORAL IMAGERY
}

\author{
Ben Gorte, Corné van der Sande \\ Delft University of Technology \\ Geoscience \& Remote Sensing \\ Stevinweg 1, 2629 HS Delft, Netherlands \\ b.g.h.gorte@tudelft.nl
}

KEY WORDS: Change detection, Orthorectification, Rendering, Radiometry, Geometry

\begin{abstract}
:
Change detection on the basis of multi-temporal imagery may lead to false alarms when the image has changed, whereas the scene has not. Geometric image differerences in an unchanged scene may be due to relief displacement, caused by diferent camera positions. Radiometric differences may be caused by changes in illumimation and shadow between the images, caused by a different position of the sun. The effects may be predicted, and after that compensated, if a $3 \mathrm{~d}$ model of the scene is available. The paper presents an integrated approach to prediction of and compensation for relief displacement, shading and shadow.
\end{abstract}

\section{INTRODUCTION}

The task addressed is validation of existing geoinformation using newer aerial imagery, whereby it is assumed that older imagery (used during creation of the information to be validated) is also available. The setting is about highly detailed, large scale topography in urban environments. Our experiments are at scales in the order of 1:1000 using aerial images of $3.5 \mathrm{~cm}$ spatial resolution.

Validation is seen as the counterpart of object based change detection. The process tests the hypothesis concerning the validity of each object in an existing database against new data. Rejected objects require further investigation eventually leading to an update. Confirmed objects, on the other hand, will remain in the database unchanged, whereas the confidence in their validity increases. Our objective is making the validation process fully automatic, in such a way that 'confirmed' objects need no further investigation. Therefore the omission error (of unnoticed changes) should be small. This usually comes at the cost of higher commission errors (false alarms), but clearly their number should be as small as possible too.

Change detection on the basis of multi-temporal imagery may lead to false alarms if (A) the scene has changed (and therefore also the image), but the changes are not relevant, or (B) the image has changed, but the scene has not. In both cases changes may be detected, although the existing information is still valid. Case (A) concerns, for example, moving cars, results of painting and gardening, falling leafs, wet vs. dry pavement etc. In the paper, however, we focus on (B).

Image differerences in an unchanged scene can be characterized as geometric or radiometric. Geometric differences are due to different camera positions, causing different relief displacements. The cure is true ortho image rectification, for which a digital surface model is required, which models, in addition to the terrain, buildings at level of detail (LOD) 1 or 2, as well as other $3 \mathrm{~d}$ objects, such as trees. Radiometric differences are caused by illumination changes due to the different dates and times of image acquisition. Assuming cloud free conditions these differences manifest themselves as shading and as shadow. Our purpose is to model these effects and try to perform radiometric normalisation of the imagery accordingly. Also this proces, like the true-ortho
Table 1: 3d Model parameters

\begin{tabular}{|c|c|}
\hline \multicolumn{2}{|l|}{ Area extent } \\
\hline $\min -\max x(\mathrm{RD})$ & $154548-154900.11$ \\
\hline $\min -\max y(\mathrm{RD})$ & $462299.28-462648.33$ \\
\hline $\min -\max z$ (NAP) & $4.26-22.81$ \\
\hline \multicolumn{2}{|l|}{ Number of triangles } \\
\hline terrain & 81576 \\
\hline curbs & 65607 \\
\hline walls & 2104 \\
\hline roofs & 9740 \\
\hline
\end{tabular}

rectification, requires a $3 \mathrm{~d}$ (surface) model describing the terrain and the objects in the scene.

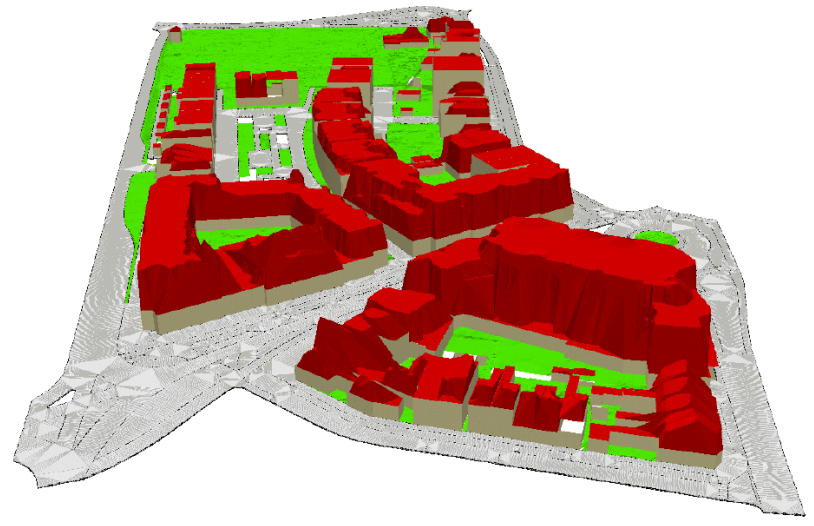

Figure 1: Automatic 3d reconstruction

We assume a DSM or 3d model to be available (Fig.1, Table 1), but part of the research is indeed about the required quality of this information for the purpose described. Note that at this stage it does not matter whether it is the same $3 \mathrm{~d}$ information is being validated, or that this information is auxillary in the course of a $2 \mathrm{~d}$ validation. In the latter case the required $3 \mathrm{~d}$ data could, for example, be generated from the (old or new) imagery by matching, or by airborne laser scanning.

Shading is the effect that the amount of direct incident sunlight 


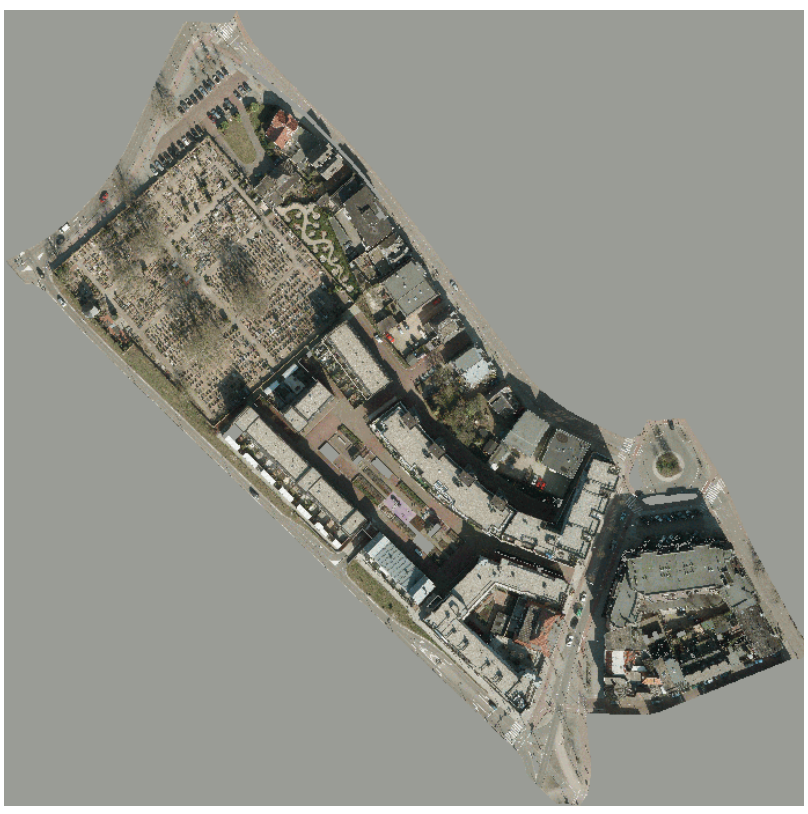

Figure 2: Masked areal image (2010)

per unit surface area depends on the sunlight direction. Assuming a (locally) flat surface, this amount is maximum when the normal vector of the surface points exactly towards the sun, and decreases with $\cos (\varphi)$ as the angle $\varphi$ between the normal vector and the sun vector increases. The amount of reflected light, as received by the imaging sensor, strongly depends on the amount of incident light. Assuming Lambertian reflectance the intensity of the reflection from a given surface patch at the sensor is independent of the sensor position. It linearly depends on the product of incident intensity and reflectivity, whereby the latter is constant in an unchanged scene. In addition to directly incident sunlight, the scene receives diffuse light, coming from all directions out of the blue sky. Its intensity at a certain surface patch is sometimes modeled as the portion of the hemispere that is visible from that patch (but we will not pursue this in this paper).

Shadow occurs if direct sunlight is not reaching certain parts of the scene, because it is blocked by other (non-transparent) objects, which are located between the sun and those shadowed parts. These parts do receive scatterred, diffuse light from the blue sky, and possibly also from reflections at other nearby objects. The indirect light that illuminates shadowed areas is indeed more blueish than direct sunlight. Note that directly-illuminated objects receive scattered light as well.

The emphasis of the paper is on modelling shadow and shading in order to get an estimate of the illumination at each surface visible in an image at the time the image was recorded. The algorithm renders a $3 \mathrm{~d}$ model, which is represented as a TIN, into a $2 \mathrm{~d}$ vertical view raster dataset with a spatial resolution matching the available imagery. A complication in our case is the size of the resulting datasets: the number of pixels of an aerial photograph is a few orders of magnitude larger than that of a computer screen. In addition, the original aerial photograph itself is true-ortho rectified using the same $3 \mathrm{~d}$ datamodel, into the same resolution as the rendering. After that it is possible to 'normalize' the images, eliminating radiometric differences from multi-temporal imagery and reduce the false-alarm rate at unchanged objects. Working 'object based' on the basis of a $3 \mathrm{~d}$ model allows for taking the accuracy and detailedness of the model, and the possible errors in shading, shadowing and orthorectification resulting from it, into account when deciding about the validity of each object.
Table 2: Aerial Photographs

\begin{tabular}{|r|r|r|}
\hline Image & 2010 & 2011 \\
\hline \hline Camera & Vexcel UltraCam-Xp \\
\hline Sensor size & $17310 \times 11310$ pixels \\
\hline Pixel size & \multicolumn{2}{|c|}{$6 \times 6 \mu \mathrm{m}$} \\
\hline Principal distance & \multicolumn{2}{|c|}{$105.0 \mathrm{~mm}$} \\
\hline Exterior Orientation \\
\hline Xo & 154697.55 & 154705.76 \\
\hline Yo & 462473.45 & 462473.90 \\
\hline Zo & 623.74 & 581.78 \\
\hline$\omega($ gon) & -0.4754 & 0.0313 \\
\hline$\varphi$ (gon) & 0.3008 & -0.1212 \\
\hline$\kappa$ (gon) & -99.166 & -99.5445 \\
\hline
\end{tabular}

Sun position determinaton

\begin{tabular}{|r|r|r|}
\hline Date & Mar 8 2011 & Apr 23 2010 \\
\hline Time of day (UTC) & $10: 27: 32$ AM & $9: 46: 21$ AM \\
\hline Longitude & E 5.38 & E 5.38 \\
\hline Lattitude & N 52.15 & N 52.15 \\
\hline Altitude & $5 \mathrm{~m}$ & $5 \mathrm{~m}$ \\
\hline Sun Azimuth angle & 59.654 & 45.366 \\
\hline Sun Zenith angle & 156.22 & 140.53 \\
\hline
\end{tabular}

\section{AVAILABLE DATA}

The $3 \mathrm{~d}$ model (Fig. 1) used in the experiment below is located in the center of the city of Amersfoort in the Netherlands. It was created from a combination of large scale $2 \mathrm{~d}$ topographic data (the Dutch BGT dataset) and a laser altimetry point cloud. The point cloud is part of the Dutch AHN-2 dataset, which was set up by the National Survey Department and the regional Water Boards, mostly for water management purposes. It is a high density (on average more than 20 pts/sq.m.) point cloud, in which terrain points and 'object points' (buildings, trees etc.) are separated, with systematic error below $6 \mathrm{~cm}$, and random error below $6 \mathrm{~cm}$ as well. The buildings in the model were reconstructed fully automatically by software of Sander Oude Elberink according to his methodology in (Oude Elberink, 2010), in the context of an urban hydrology project (Verbree e.a. 2013). The streets, including curbstones, play a major role in urban hydrology; therefore they are represented with great detail (see Table 1). The sub-model used here is located in a $350 \times 350 \mathrm{~m}$ square, which, however, is not fully covered by the model (Fig. 2).

The two aerial photographs used in the experiments cover the area of the $3 \mathrm{~d}$ model. They are part of yearly campaigns flown over the Netherlands, in this case from 2010 and 2011. Red, green and blue channels are available; the ground resolution is about $3.5 \mathrm{~cm}$. The $350 \mathrm{~m}$ area extend of the $3 \mathrm{~d}$ model, therefore, require images size of around $10000 \times 10000$ pixels.

\section{ALGORITHMS}

The goal of the following section is to remove the effects of relief displacement, as well as of shadow and shading, from aerial images, in order to make these images better comparable in a change detection context.

Both of these efforts require the availability of a $3 \mathrm{~d}$ model of the scene representing terrain and objects. As the $3 \mathrm{~d}$ model is a vector model with triangulated surfaces (see Table 1), it will turn out that the efforts involve several TIN to grid conversions, 


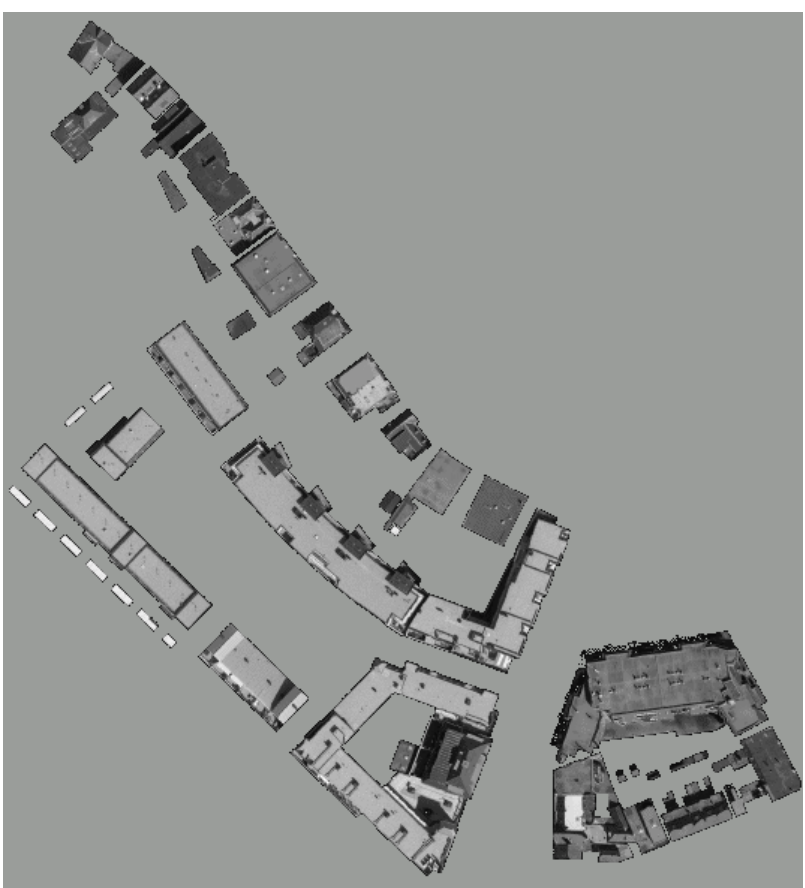

Figure 3: Building (roof) objects 2010

which will be generally described here, before going to the details of the algorithm. TIN to grid conversion is the projection of a $3 \mathrm{~d}$ triangulated model onto a $2 \mathrm{~d}$ plane, sampled regularly into a grid. The sampling space in the model coordinate system determines the grid resolution. The process works as follows: first, an empty grid is constructed on the basis of the size of the projected area (the minimum and maximum $\mathrm{X}$ and $\mathrm{Y}$ coordinates) and the wanted resolution. Next the process works one triangle at a time. A bounding box around the projected triangle determines the candidate grid cells for this triangle. Which grid cells are actually involved is decided by submitting each of them to a point-in-triangle test.

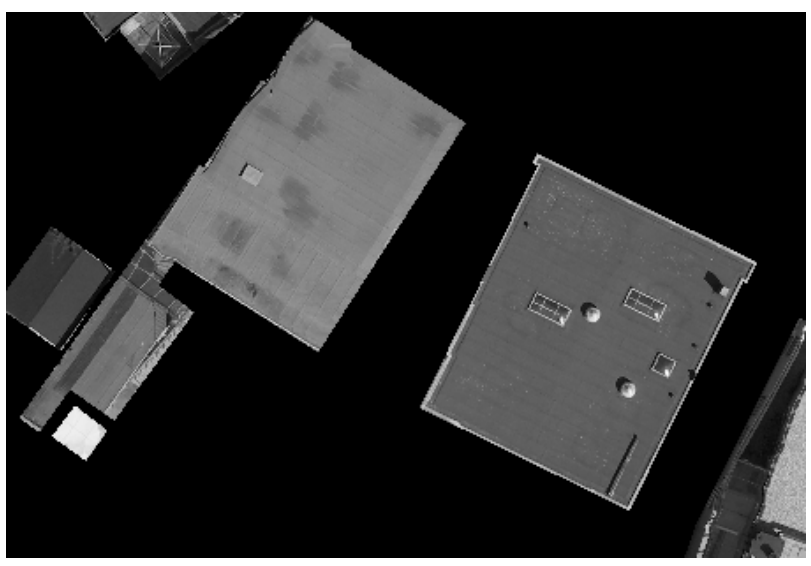

Figure 5: Roofs (detail) 2010

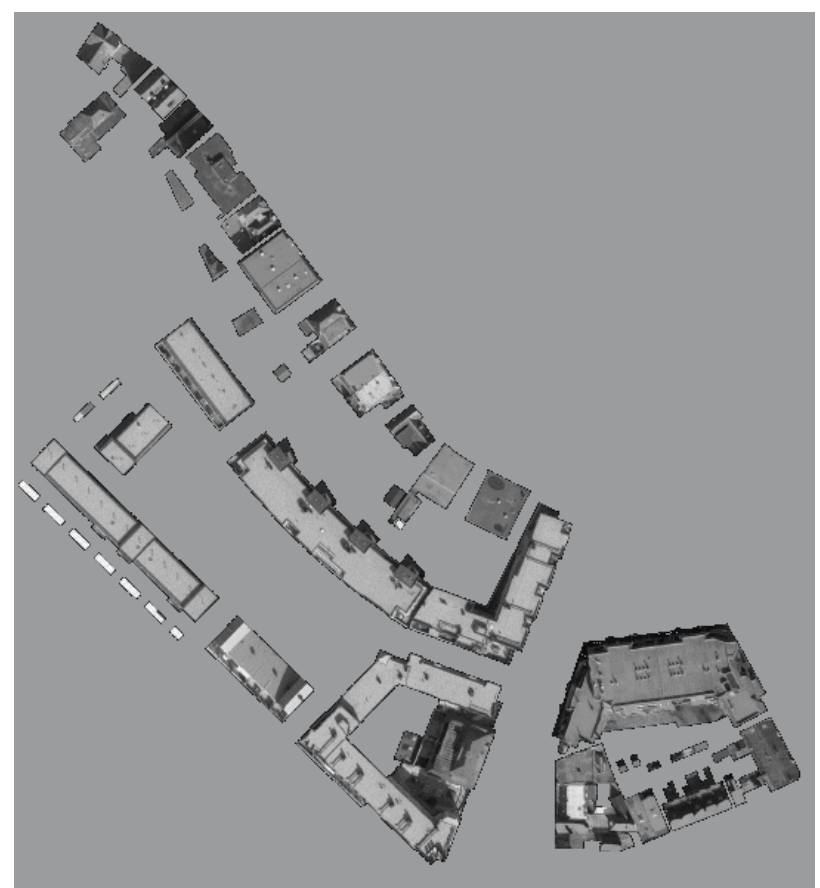

Figure 4: Building (roof) objects 2011

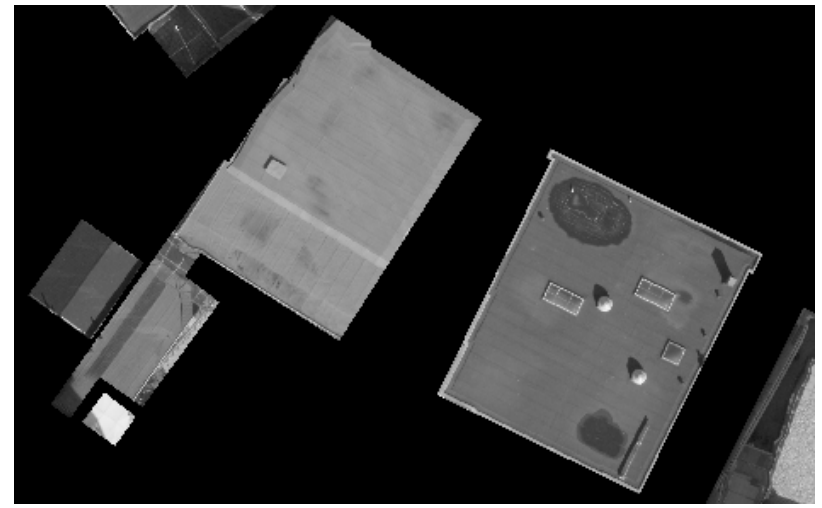

Figure 6: Roofs (detail) 2011

Also $3 \mathrm{~d}$ rendering, which is the visualisation of a triangulated $3 \mathrm{~d}$ model into an image on a computer screen, can be considered TIN to grid conversion. A camera position is assumed inside the model. This is the projection center, and the projection plane is perpendicular to the camera axis at a distance $f$ (the focal distance of the camera) from the projection center. The grid positions will correspond to the image pixels. While doing the conversion for a certain triangle the distances between the selected grid cells and the camera are computed. It will occur that a single grid cell is involved in the conversion of more than one triangle. Now only the triangle closest to the camera should influence the rendering; all the others are "hidden". In a so-called Z-buffer this distance is recorded for every grid position. If a closer triangle comes along the Z-buffer is updated; results for farther triangles are simply discarded.

The content of the pixels (in a rendering context), is determined by the reflection of incident light off the projected surfaces towards the camera. Note that the light sources, in terms of their location and light properties, should be specified, in addition to the camera position. 


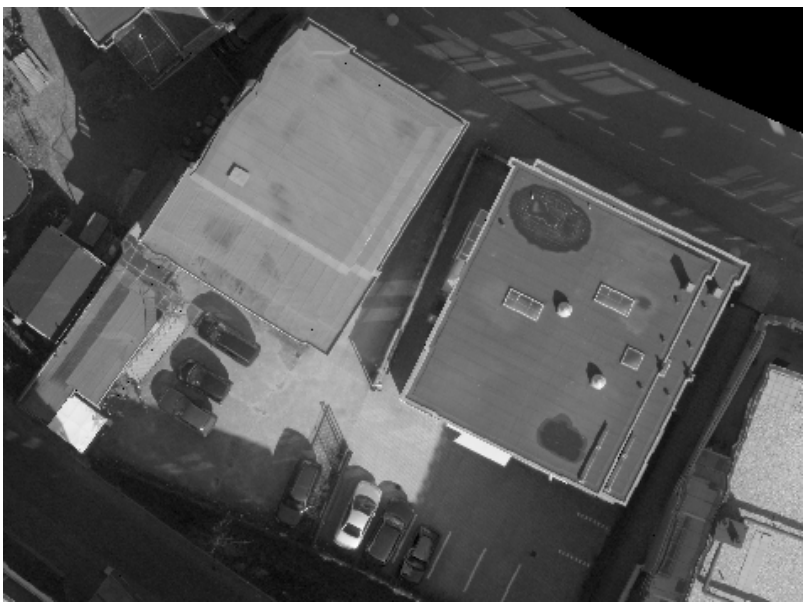

Figure 7: True ortho (detail) 2011; note the double roof edge at the building to the right, due to occlusion.

\subsection{True-ortho rectfication}

Change detection will be carried out in an object based fashion: each object in the geo-database is considered a hypothesis, and is evaluated against the image content. Of particular relevance is the question whether the support for an hypothesis has decreased between the older and the newer image. Therefore it is necessary that an object is covered by exactly the same portion of either image, irrespective of the object being at terrain level (e.g. streets, gardens, parking lots) or above terrain (e.g. buildings, tree crowns)

The standard way to accomplish this with areal images is by trueortho rectification. This is a vertical projection of the TIN 3d model onto the $(\mathrm{X}, \mathrm{Y})$ plane, which is then sampled into a regular grid. The projection center is at infinite height. For each grid cell, sampled at coordinate $(\mathrm{Xp}, \mathrm{Yp})$ in the projection plane, the $\mathrm{Zp}$ value can be found, according to the model, at the surface that is visible in the image. By using the exterior orientation parameters of the aerial image the pixel position in that image is known, and the value at that pixel can be copied into the current grid cell of the ortho image.

When using images of different epochs in a change detection context, the above will ensure that the correct sets of pixels will be assigned to each object. It is possible the generate a grid for the entire model (all visible objects), or for selected objects only. In Fig. 3 and 4 only roof objects, as seen in both images, are shown, and can be compared one by one in a change detecton algorithm. A detail of both images is show in Fig. 3 and 3. Note that at certain $(\mathrm{X}, \mathrm{Y})$ positions the "top" object in the model is not visible in the image due to occlusion. There, the value in the ortho-image is wrong, as in Fig. 7. The entire roofs are correct, but the occluded part of the terrain immedately next to the building is shown incorrectly (by repeating the roof pixels); the alternative would have been to put the unknown value at those places.

\subsection{Shadow and Shading}

Predicting shadow and shading in an ortho image of a $3 \mathrm{~d}$ model can be described as a TIN to grid conversion as well. The pixel value at a certain grid position is now determined by the question whether that position receives direct sunlight. If the answer is no, it is a shadow pixel, and if it is yes, the incidence angle of the sunlight onto the surface facet given by the triangle in the model determines the shading.

The process knows the position of the sun during the image recording from the lat-long location of the scene and the data and time

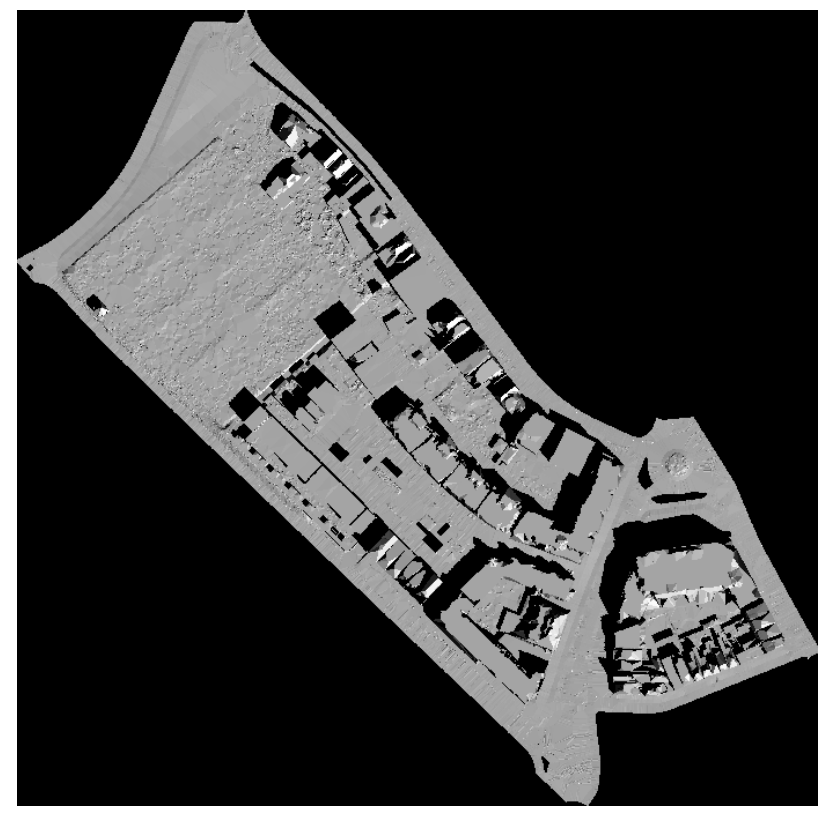

Figure 8: Simulated shade and shadow 2010

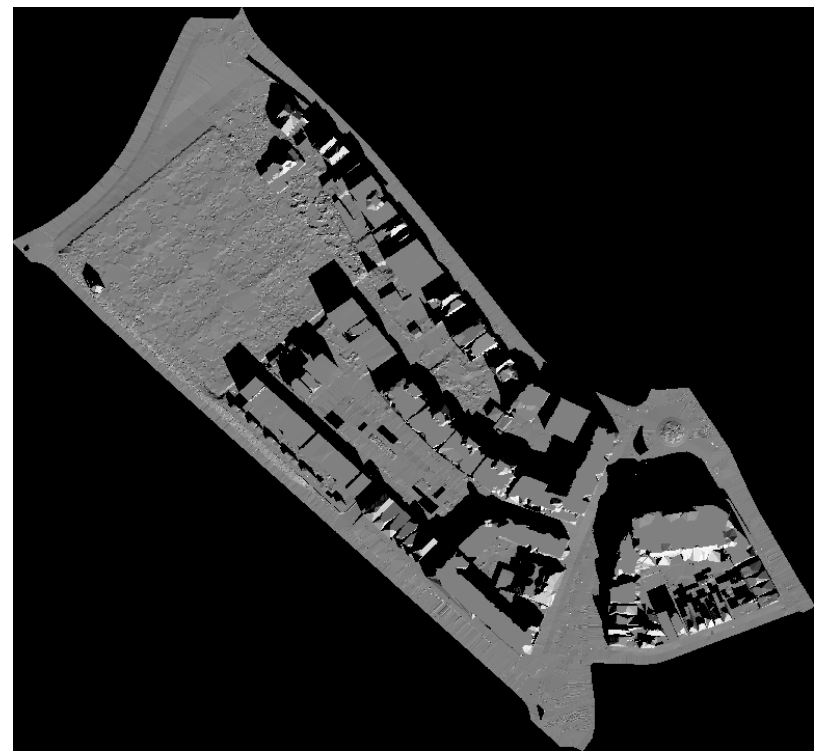

Figure 9: Simulated shade and shadow image 2011

of the acquisition. We compute this position (i.e. the azimuth and zenith angles) using the algorithm of Reda and Andreas (2003) in the implementation of Vincent Roy (2009), made available through the Matlab Central.

Introducing cast shadows into rendering is a non-trivial, however solved, problem in computer graphics. Chris Bentley (1995) describes two alternatives, (a) using Z-buffering (Algorithm3.2) and (b) using Ground transformation. Our algorithms is modified Zbuffering (Williams, 1978), having both camera and light source at infinite distance (Blinn 1988).

As in Algorithm 3.2, the process has two phases. During phase 1 the solar angles are used to rotate the model such that it can be rendered with the camera at the Sun's position. A grid is created by Z-buffering to find out which parts of the model are "visible" from the Sun, in order to reveal that the remainder of the model is in the shadow. During phase 2, rendering is done from a projection center at the real camera position, as usual, but at the same time the (now rotated) "rendering result" of phase 1 is used to add 


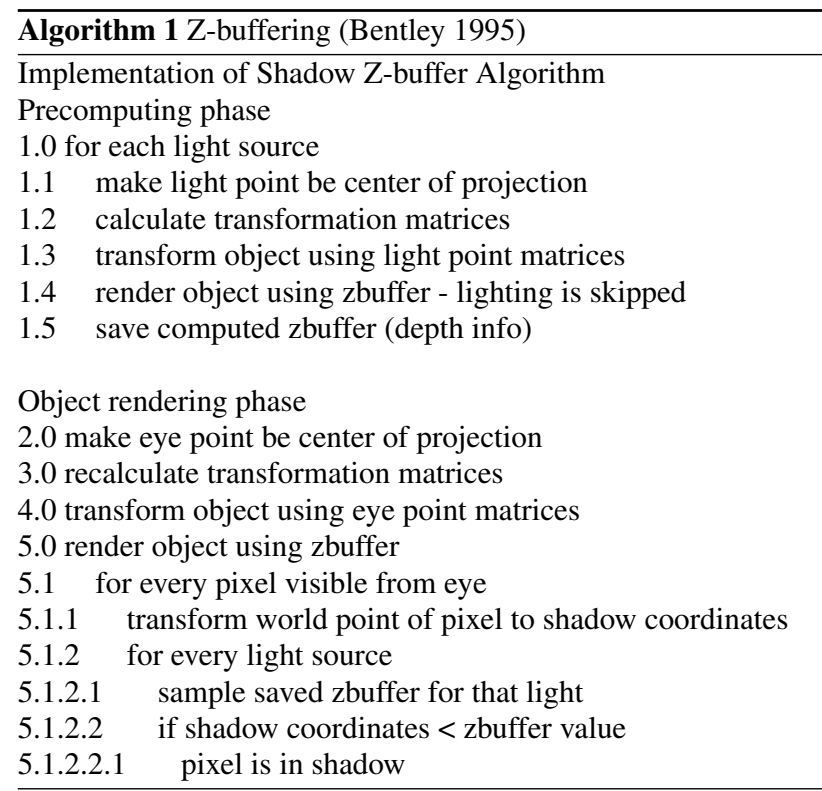

shadow information.

A benefit is that shading information is obtained "on the fly" when modelling shadow: in the rotated model of phase 1 the angle between the normal vector of a triangle (from the plane equation given by the three vertices) and the sun vector, which equals $(0,0,1)$, is easily computed and stored with the triangle list. During phase 2 the cosine of this angle is used as the output value in the shading image. Note that shading computation at non-flat building roofs requires an LOD2 building model.

\subsection{Shadow and shading compensation}

Sun-lit surfaces in the scene receive both direct and indirect (diffuse) illumination. Radiation from the sun in cloud-free wheather circumstances partly travels to the earth surface in straight lines (if we ignore refraction). Another part is absorbed by the atmosphere, and yet another is scattered. The effects of absorption and scattering are wavelength-dependent. Absorption happens at very specific wavelengths, called absorption bands. Scattering, including the Raleigh scattering considered here, is a broad-band phenomenon; it is stronger, however, as wavelengths get shorter, and therefore in the visible range of the spectrum blue light is scattered more than green and red (to mention just a few). Therefore the sky is blue and, moreover, the illumination at shadowed parts of the scene is, apart from being weaker, contains a relatively large amount of 'blue', compared to the solar spectrum. Consequently, in the directly-illuminated parts of the scene the light contains a slightly larger (relative) amount of green and red, compared to the solar spectrum (which is rarely corrected for, however).

The purpose of modelling shadow, in this paper, is to find out which pixels in the true-ortho rectified image are directly illuminated and which ones are in the shadow. If we take the former ones as 'correct', the shadow effect at the latter can be compensated by multiplying their pixel value by a factor that depends on the estimated ratio between direct and diffuse illumination. In order to account for wavelenth dependency, different factors could be applied for the red, green and blue image channels. Thanks to the excellent radiometric resolution of nowaday's digital cameras also shadowed parts of the scene have sufficient contrast to make this multiplication work.

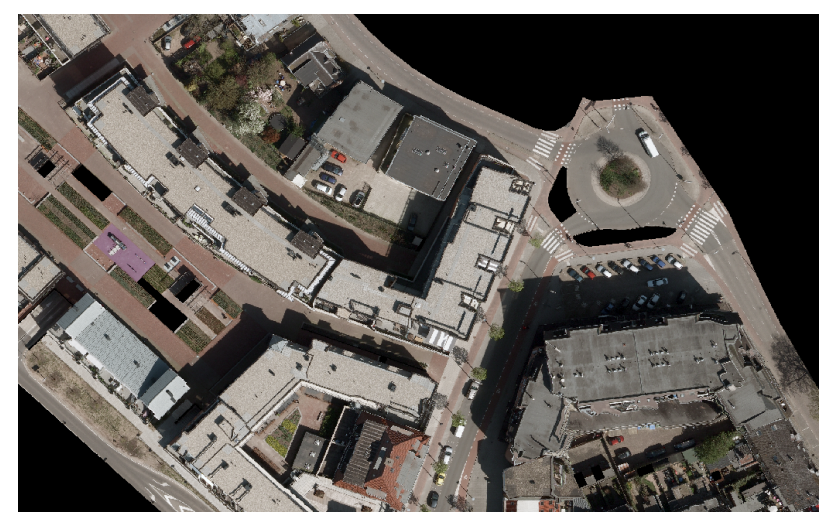

Figure 10: Ortho image 2010

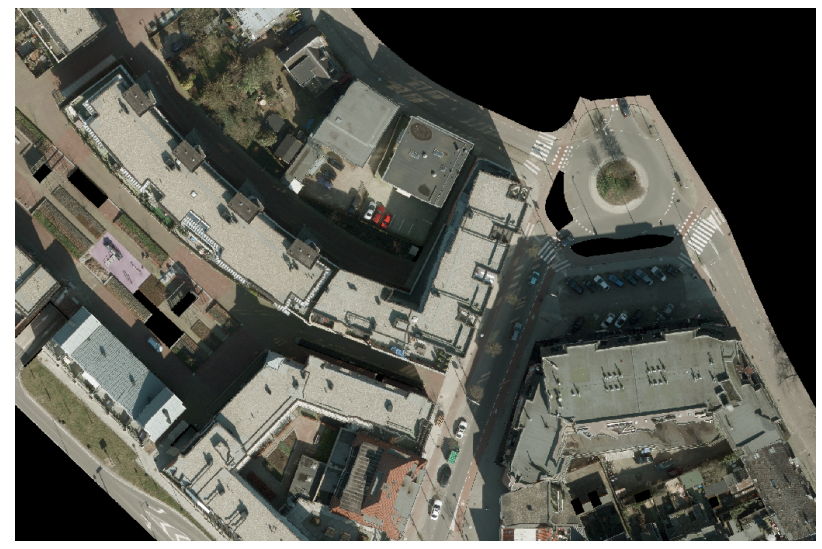

Figure 11: Ortho image 2011

\section{Shading}

The amount of direct illumination at sun-lit surfaces is proportional of the cosine of the angle between the surface normal and the sun vector. With a zero-angle (the surface being perpendicular to the solar rays), the intensity is maximum, gradually going down to 0 as the angle tends to 90 degrees. The cosine was computed during phase 1 of the shadow algorithm, and stored in the shading image during phase 2 .

The amount of diffuse illumination, in a first approximation, can be considered the same in all cases, as diffuse light is coming from all directions. Therefore, the total illumination at a surface can be computed as a constant (for the diffuse component) plus a variable amount, as given by the shading image. The constant is equal to the factor, applied above, for shadow compensation - the shadows have value 0 in the shading image.

\section{RESULTS AND DISCUSSION}

The above-described algorithms for true-ortho rectification and shadow/shading estimation and compensation are applied to the two Amersfoort images of 2010 and 2011. The variable amount of direct illumination (on a scale of 0-255) was estimated in shading images (Fig. 8 and 9). The shadowed areas in those images have value 0 (black). Fig. 8 has a generally brighter appearance than Fig. 9. This is not an error, as the corresponding aerial images are from end of April vs. beginning of March. Therefore the sun is higher in the first, leading to more intense illumination. On the same scale the amounts of (constant) diffuse illunination was estimated as 80, 90 and 100 for red, green and blue respectively.

The overall effect becomes visible in Fig. 12 and 13, which clearly show less shadow than the true-orthos shown in Fig. 10 


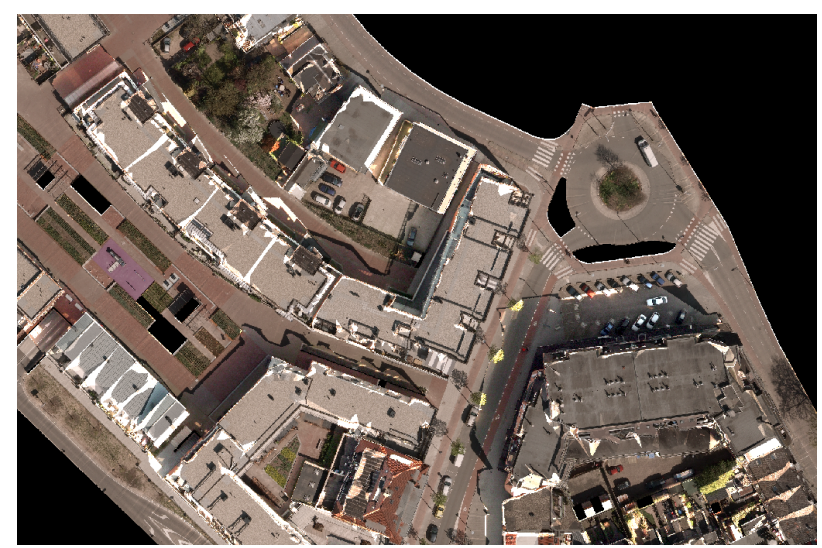

Figure 12: Shading/shadow compensation 2010

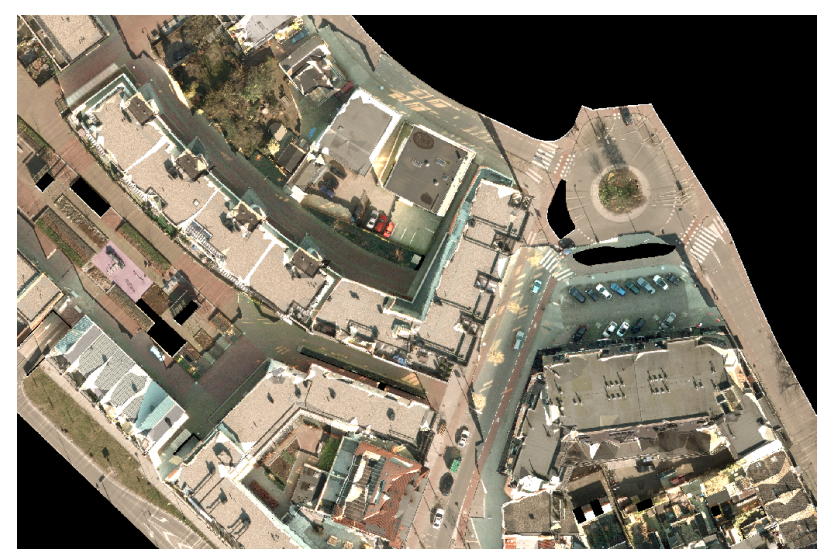

Figure 13: Shading/shadow compensation 2011

and 11. Unfortunately also some defects are visible, such as the bright areas to the North and East of some of the buildings. These are caused by incorrectly handling the effect of occlusions mentioned earlier. Also there are some sun-lit tree crowns above shadowed parts of the terrain surface; those tree crowns are incorrectly 'compensated'. Conversely, shadows cast by trees are not modelled, as there are no trees in the model. Therefore compensation at these shadowed areas did not take place.

\section{CONCLUSION}

The paper presents a integrated method to account for effects occuring in areal imagery due to the $3 \mathrm{~d}$ nature of urban scenes. These effects are relief displacement, shadow and shading. To consider those effects is highly relevant during change detection, as they may manifest themselves differently in subsequent images, due to differences in camera position and Sun position. This may lead to false detection of changes. Increasingly available $3 \mathrm{~d}$ information in urban environments may be used to predict those effects, leading to the opportunity to compensate for them on the basis of estimates of the ratios between direct and diffuse illuminations, also depending on the wavelength.

The next step in our research will be to investigate whether the corrected imagery indeed leads to improved change detection. Meanwhile we will study the extend to which the quality of $3 \mathrm{~d}$ models influences the results. Lastly, we want to include other objects than buildings, such as trees, in the approach.

\section{REFERENCES}

Bentley, Chris (1995), Two Shadow Rendering Algorithms, http://web.cs.wpi.edu/ matt/courses/cs563/talks/shadow/ shadow.html

Blinn, James (1988), "Me and my (fake) shadow", IEEE Computer Graphics and Applications, January 1988.

Maren, Gert van and Jinwu Ma (2012) , 3D Analyst - Feature \& Volumetric Analysis, Esri International User Conference, San Diego, July 2012.

Oude Elberink (2010), S, Acquisition of 3D topography: automated 3D road and building reconstruction using airborne laser scanner data and topographic maps, $\mathrm{PhD}$. Thesis, University of Twente, 2010.

Reda, I., Andreas, A. (2003) Solar position algorithm for solar radiation application. National Renewable Energy Laboratory (NREL) Technical report NREL/TP-560-34302.

Roy, Vincent (2009) , http://http://www.mathworks.com/ matlabcentral/fileexchange/4605-sunposition-m, accessed 28 March 2014.

Verbree, E, M de Vries, B Gorte, S Oude Elberink, G Karimlou (2013), Semantic 3D city model to raster generalisation for water run-off modelling, SPRS Annals of the Photogrammetry, Remote Sensing and Spatial Information Sciences, Volume II-2/W1.

Williams, L (1978), "Casting Curved Shadows on Curved Surfaces", Computer Graphics, vol. 12, no. 3, pp270-4, 1978. 